\title{
Study on the Test Scheme of Ball Screw Dynamic Characteristic Parameters
}

\author{
Haitao $\mathrm{Li}^{1, \mathrm{a}}$, Junjie Guo ${ }^{1, \mathrm{~b}}$, Hongfeng $\mathrm{Du}^{1, \mathrm{c}}$, Jindong Wang ${ }^{2, \mathrm{~d}}$ \\ ${ }^{1}$ School of Mechanical Engineering, Xi'an Jiaotong University, Xi'an 710049, China \\ ${ }^{2}$ School of Mechanical Engineering, Southwest Jiaotong University, Chengdu 610031, China \\ apirate328@163.com, bjunjieg520@163.com, ${ }^{\mathrm{c}}$ dhfmailbox@163.com, ${ }^{\mathrm{d}}$ wangjindong198205@163.c \\ om
}

Keywords: ball screw; dynamic characteristic parameters; test bed; test scheme.

\begin{abstract}
The dynamic characteristic parameters of ball screw includes positioning accuracy, repeat positioning accuracy, speed and acceleration, friction torque, preload, fatigue properties, temperature rise and so on. In this paper, a new multi-function ball screw measuring device is developed. The majority dynamic characteristic parameters of ball screw can be tested on it by the means of simply assembly or dismounting related sensors and testing software. This paper describes the building of test bed and test schemes in measuring different parameter in details.
\end{abstract}

\section{Introduction}

In recent years, with the improvement of modern manufacturing technology, mechanical equipments like numerical control machine tool (CNC machine) and robot have faster feed rate, which is making progress of ball screw assembly toward high speed ${ }^{[1][2]}$. At the present stage, there is large gap in quality of rolling components between our country and developed country. Meanwhile, the partial defect of testing instruments and equipment and lack of unified inspection procedures in mechanical industry hinder the development of theoretical research and production practice ${ }^{[3,4,5]}$. Aiming at the solution of the problems above mentioned, the multi-function ball screw measuring device is developed to meet the requirements of high-performance CNC machine. The multi-function ball screw measuring device can test the majority dynamic characteristic parameters of ball screw like positioning accuracy, repeat positioning accuracy, speed and acceleration, friction torque, preload, fatigue properties, temperature rise and so on ${ }^{[6][7]}$. It can give the ball screw a comprehensive and reliable evaluation with low cost.

\section{The test bed and test scheme}

Test bed of positioning accuracy and repeatability positioning accuracy.Measurement principle and characteristics. Positioning accuracy of ball screw assembly refers to the axial dynamic deformation including the lead error, in other words, it is the errors by positioning at any point within the scope of the trip. It has close relationship with lead error, contact deformation between ball and railway surface because of preload or axial force, deformation caused by temperature variation, the ways of supporting and rigidity of nut seat. In fact, positioning accuracy refers to the displacement error, it is the difference between theoretical displacement and actual displacement of ball screw assembly. Therefore, we choose the circular grating encoder and the long grating encoder to build the positioning accuracy measurement system. The long grating encoder detects the actual axial displacement, and the circular grating encoder measures the rotation angle of ball screw ${ }^{[2][4]}$.

The linear motor loads the axis force to simulate the actual conditions. The size of the loading force can be controlled by computer programs. Compared with the hydraulic loading, it has the characteristics of less space, faster response and no pollution. Compared with the counter weight loading, it has smaller volume, greater acceleration. The ball screw nut preload can be regulated by round nut which will be introduced in details in part 2.2. 
The ball screw moves in circle, and nut moves in axial direction driven by AC servo motor. The location information of both can be read by the circular grating encoder and the long grating encoder, we symbol them as $\theta$ and $S$. So the positioning error can be calculated by the formulation: $\Delta=S-P * \theta / 2 \pi$.

Structure design of positioning accuracy test bed. As is shown in figure 1 , it is the structure design diagram of positioning accuracy test bed. The ball screw is supported by front bearing seat 3 and back bearing seat 12 at both ends. The AC servo motor 11 is fixed on the tailstock slider 13 to transmit power to ball screw 5. It forms close-loop with circular grating encoder 1 to achieve high-precision positioning. The nut 10 and nut seat 9 is driven by ball screw, grating reading head fixed on them feeds back the actual location information to computer system ${ }^{[1][8]}$.

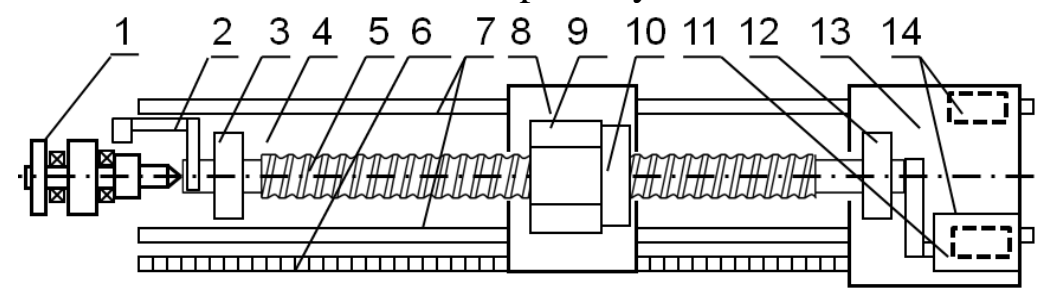

1 circular grating encoder 2 fork 3 front bearing seat 4 linear motor 5 ball screw 6 long grating encoder

7 linear guide ways 8 linear guide slider 9 nut seat 10 nut 11 AC servo motor 12 back bearing seat

13 tailstock slider 14 pneumatic clamps

Fig.1 Structure design diagram of test bed

Preload changeable friction torque test bed. Currently, the research on friction torque of ball screw is still in blank field in influence factors, fluctuation reasons, reasonable lubrication parameters and noise principle and so on. In China, there is not yet unified cognition about these problems. The most conspicuous features of friction torque of ball screw are imbalance, large fluctuations and bad repetitiveness. That is because too many factors have influence on it like the structure of ball screw assembly, machining precision, preload, internal lubrication, external environment and operating state. The preload has strong influence on friction. Cut down the preload, the friction energy loss decreases sharply, and system calorific value becomes small, wear decreases, the fatigue life increases. On the contrary, increasing the preload will improve the system stiffness and reduce the empty return error. In order to study the quantitative relations of them, a set of complete test system is essential $^{[6]}$.

Measurement principle and features. Double force sensors are used to measure the friction torque rotating in forward and reversal direction. As shown is figure 2, the double force sensors are fixed on the base which can move in axial direction. The measuring bar is fixed on the nut. The bottom of measuring bar is stuck in the middle of double force sensors, and the force value can be adjusted by pressing plates. When measuring, the ball screw rotates at constant velocity, the nut can't rotate but move in axial direction. When the ball screw rotates in clockwise, read the relative changes in value of the left force transducer, on the contrary, read the relative changes in value of the right force transducer. Then, the friction torque can be impressed as: $T_{S}=\Delta F * L=\left(F_{1}-F_{0}\right) * L$. In this equation, $T_{S}$ refers to the friction torque being measured, $\Delta F$ refers to the relative changes in value of force transducer, $L$ refers to distance between the measuring point and screw axis, $F_{0}$ refers to the initial value of force transducer, $F_{1}$ refers to final value of force transducer ${ }^{[1]}$. 


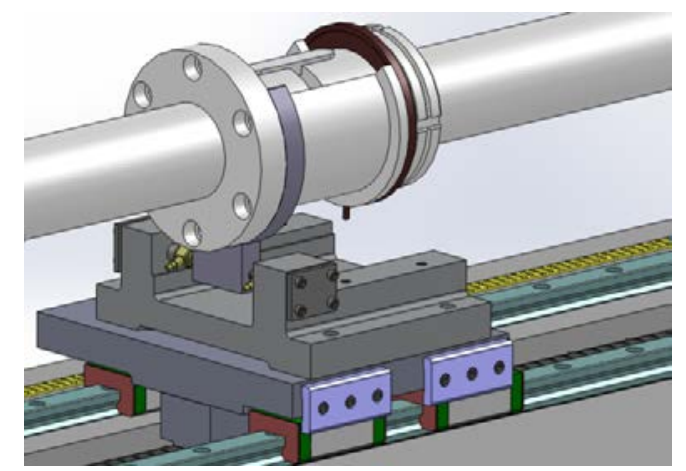

Fig.2 3D modeling of friction torque test bed

In order to measure the friction torque imposed on different preload, pressure sensor is added between sleeve and round nut, and the preload can be adjusted by the round nut. The preload value can be read by the pressure sensor and sent to the computer system.

Before measurement, adjust the preload and clamping force between two force sensors to required value in advance, and record the initial value. When measuring, the linear motor drives the base move in axial direction, meanwhile, the AC servo motor drives the nut and measuring bar move in axial direction, too. Record the relative changes in value of the two force sensors, then, the friction torque can be calculated by the formulation above mentioned.

Structure design of friction torque test bed. As is shown in figure 3, the base and the linear guide ways slider are locked together and driven by linear motor. The drive system forms full close-loop with the long grating encoder. Similarly, the measuring bar and nut are driven by AC servo motor forming semi-closed loop with circular grating encoder. The two parts of measuring unit are driven by different drive system, so there is a displacement error between them. In order to solve this problem, a displacement error elimination device is set between force sensors and measuring bar, or the force sensors may be damaged in measurement. The ball slot is mounted on force sensors, the ball is put in the slot, and there is elastic material filled in the blanks between them. Therefore, the mini-displacement generated by the two parts of the device can be eliminated ${ }^{[8]}$.

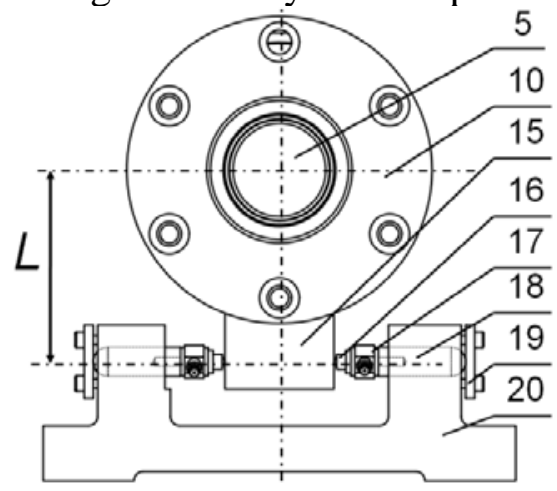

(a)

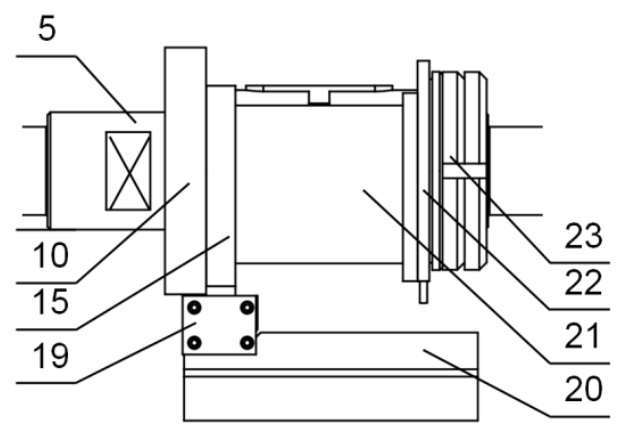

(b)

5 ball screw 10 ball screw nut 15 measuring bar 16 ball slot 17 force sensor 18 small pillar19 pressing plate 20 base 21 socket 22 pressure sensor 23 round nut

Fig.3 Structure design diagram of friction torque test bed

The structure design above-mentioned (drive the two parts respectively) can eliminate extra axial force generated by the way of "nut drives the linear slider". In this way, the measured value of friction torque just has relationship with the preload, it facilities the data processing.

Fatigue life test bed. Ball screw has several common failure modes like surface damage, large deformation, balls broken and so on. With the condition of normal installation, lubrication and normal use, the primary failure mode is fatigue failure, namely, it is the fatigue pitting of ball, ball screw and nut groove. Therefore, fatigue is the main factor effecting the service life. In order to study the relations between parameters of ball screw and fatigue life, relevant research and test bed are necessary. 
Measurement principle and features. The test bed is made up of two parts: mechanical system and measurement \& control system. The mechanical system includes base, drive units, load unit, ball screw being measured and auxiliary unit. The measurement \& control system includes AC servo system, linear motor intelligent load unit, detecting unit and industrial personal computer (IPC). Controlled by the IPC, the linear motor loads to ball screw being measured according to preset procedures, and conducts the fatigue life test by continuously reciprocating motion. The detecting unit working closely signal recognition software can detect the fatigue rupture of ball screw, and decide the terminate time. Meanwhile, the system has perfect functions of test data save, management and statistical analysis.

Speed and acceleration test. The acceleration measurement and study of high speed ball screw can improve the characteristic of acceleration and deceleration, improve the ability of quickly tracking, decrease the instantaneous elastic deformation when start and stop, improve the positioning accuracy and repeat positioning accuracy of ball screw at high speed. In the acceleration measurement system, the acceleration sensor is fixed on the workbench in order to decrease the vibration effects from ball screw moving at high speed. The figure 4 shows the installation of acceleration sensor.

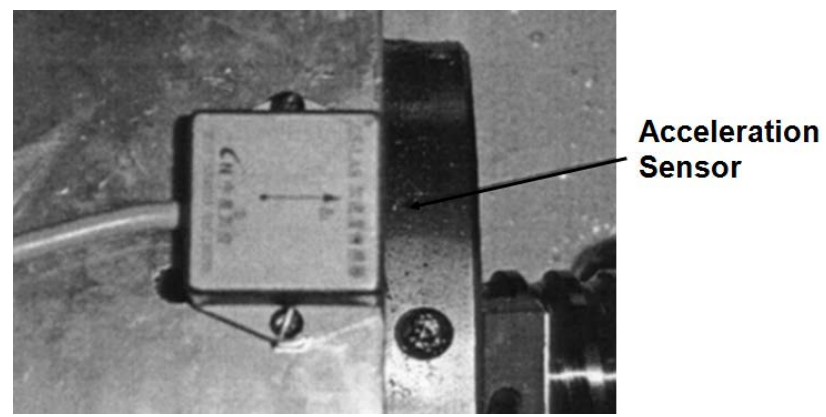

Fig.4 Installation of acceleration sensor

The acceleration measurement system is made of acceleration sensor, data acquisition card, industrial personal computer. It can conduct the acceleration signal collection, curve display, data analysis and print by software programming. The figure 5 shows the structure diagram.

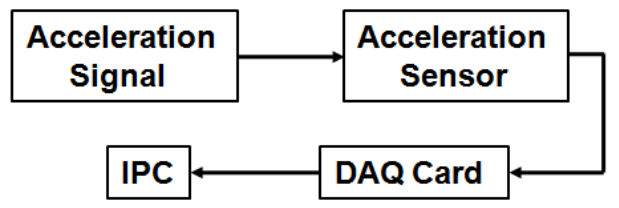

Fig.5 structure diagram of acceleration measurement system

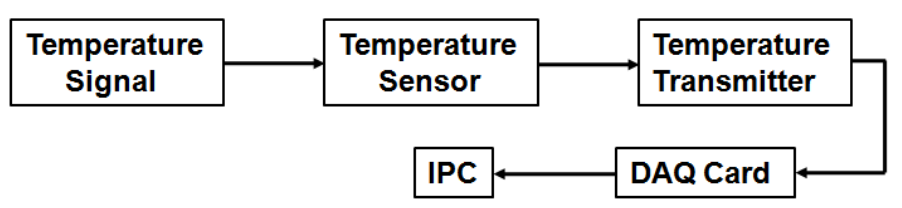

Fig.6 Structure diagram of temperature measurement system 


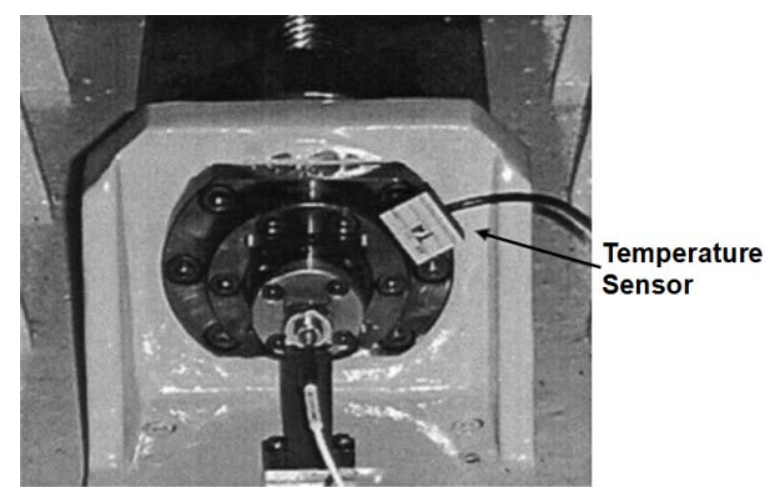

Fig.7 Installation of temperature sensor

Temperature rise test. In the process of ball screw high speed, the problems of noise, temperature rise and thermal deformation have to be solved. Related experiments shows that the noise intensity increases 4-5decibel, and the nut temperature rises 5-6 ${ }^{\circ} \mathrm{C}$ for every $1000 \mathrm{r} / \mathrm{min}$ increase if no measures decreasing the noise and vibration have been taken. Meanwhile, the temperature rise leads to the thermal deformation which reduces the positioning accuracy of whole measurement system. Therefore, temperature rise measurement system is an important part of ball screw integrated test system. The temperature variation of bearing seats at both ends, nut is more obvious than other parts, therefore, there are three sensors set on the test bed to measure the three parts.

The temperature measurement system is a typical data acquisition system. It consists of temperature sensors, data acquisition card, and industrial personal computer. It can conduct the temperature signal collection, curve display, data analysis and print by software programming. The figure 6 shows the structure diagram. The figure 7 shows the installation physical map of temperature sensors.

\section{Conclusion}

This paper has introduced a new multi-function ball screw measuring device, and the measurement principle, the building of test bed, the experiment process and test software for different parameter are given in details. It can give the ball screw a comprehensive and reliable evaluation with low cost.

\section{Acknowledgements}

This research has been supported by China Technology Major Project (NO: 2011ZX04016-031).

\section{References}

[1] Feng Hu-tian. Comprehensive performance measurement method and technology of ball screw Mechanism [M]. Beijing, Mechanical Industry Press, 2011:15-58.

[2] Liu Jie-liang. Analysis and Experiment Study on Dynamic Performance of Ball Screw Mechanism [D]. Master Thesis. Xi'an University of Technology, 2005: 8-18.

[3] Mou Shi-gang, Research on Dynamic characteristic of high-speed Ball Screw with nut Driven [D]. Doctoral Thesis. Shandong University, 2013.

[4] Song Xian-chun, Liu Jian, Wang Zhao-tan, Liu Xian-yin, Li bao-min. Research and Development of Test System of Combination Property of High-Speed Ball Screw Unit [J]. Tool Engineering. 2005,39(3).

[5] Wang Zhao-tan, Liu Xian-yin, Li Bao-min. Test Instrument for Comprehensive Performance of High Speed Precision Ball Screw Units [J]. Manufacture Technology \& Machine Tool. 2007, (5). 
[6] Kang Xian-min, Fu Wei-ping, Wang Da-cheng, Wang Jian-sheng. Analysis and Experiment of Effect of Preload Force on Ball Screw Friction Torque Fluctuation [J]. Journal of Mechanical Transmission. 2010, 34(6).

[7] Kang Xian-min. Analysis and Experiment Study on Dynamic Performance of Ball Screw Mechanism [D]. Doctoral Thesis. Xi'an University of Technology. 2011.

[8] Zhang Shan-zhong. Precision Instrument Structure Design Manual [M], Beijing, Mechanical Industry Press,2009: 474-497. 